\title{
Quality improvement in trauma care
}

\author{
Ingo Marzi
}

Received: 31 December 2012/ Accepted: 4 January 2013/Published online: 30 January 2013

(c) Springer-Verlag Berlin Heidelberg 2013

Improvement of quality in the care of multiple trauma patients is an ongoing issue at the various setting (e.g., prehospital setting, emergency room, surgery, intensive care, and rehabilitation). We have recently published a variety of issues focusing on this field, and particularly on quality control in trauma registries [1]. Trauma registries around the world are currently in deep discussions aimed at identifying common definitions and quality parameters, and we are curious to see the first results of those discussions. In this issue, we focus on quality improvement of prehospital trauma care and possible structural changes in the future. In addition, we look at diagnostic approaches during initial emergency room care.

Aubuchon et al. [2] from the Netherlands compared the prehospital care received by patients with severe traumatic brain injury today with that provided twenty years ago and found similar outcomes, despite the fact that the time at each scene had changed. Today, the time at the scene is almost four times as long as it was twenty years ago. This unexpected result demonstrates that traumatic brain injury remains one of the most challenging diagnoses to improve in the future. In this respect, Lansik and Leenen [3] performed a very thorough review of polytrauma care in the Netherlands. They came up with the concept that most severely injured patients would optimally be concentrated in high-volume trauma centers, including a well-organized prehospital system (e.g., helicopter, emergency physicians). Such centers could work closely with level II and III centers, allowing a well-defined rescue system.

I. Marzi $(\bowtie)$

Department of Trauma Surgery,

University Hospital, 60590 Frankfurt, Germany

e-mail: marzi@trauma.uni-frankfurt.de
Upon arrival at a trauma center, various well-defined diagnostic procedures take place according to the guidelines for trauma care (e.g., the updated S3 guideline in Germany: http://www.awmf.org/leitlinien/detail/11/012019.html). Ultrasound is one of the procedures that is performed within the first few minutes after arrival [4]. While surgeons perform FAST (focused abdominal ultrasound in trauma) in most countries, radiologists are in charge of this in some settings. Tajoddini et al. [5] compared the quality of ultrasound performed by these two groups of physicians in cases of blunt abdominal trauma in a prospective manner. They demonstrated that both groups provided comparable diagnostic specificities considering the requirements of the emergency setting.

In addition to ultrasound, CT scanning has found widespread use for the primary diagnosis of multiple trauma patients, leading to a better outcome [6]. However, the hazards of radiation need to be discussed, and further improvements in CT scanning technology and protocols are on the way. Kepros et al. [7] reviewed the residual risks of ionizing radiation very thoroughly with respect to trauma patient care. Accordingly, further prospective studies are warranted in order to determine the optimal application of this helpful procedure.

Lastly, a well-performed study by Dübendörfer et al. [8] from Switzerland prospectively evaluated the role of serial lactate levels in patients with and without traumatic brain injury. They demonstrated that lactate is a strong indicator of the development of sepsis as well as outcome in multiple trauma patients. Lactate was less relevant in isolated traumatic brain injury patients, but the SOFA organ failure score differentiated between survivors and non-survivors following isolated traumatic brain injury.

Overall, outcome of trauma care has substantially improved during the last decade, due to various 
circumstances. However, further improvements in trauma care remain an ongoing task and appear possible in relation to organization, concentration of trauma care, and technical and laboratory procedures.

Conflict of interest None.

\section{References}

1. Lefering R, Ruchholtz S. Trauma registries in Europe. Eur J Trauma Emerg Surg 2012;38:1-2.

2. Aubuchon M, Hemmes B, Poeze M, Jansen J. Prehospital care in patients with severe traumatic brain injury: does the level of prehospital care influence mortality? Eur J Trauma Emerg Surg. doi:10.1007/s00068-012-0218-6.

3. Lansink KWW, Leenen LPH. History, development and future of trauma care for multiple injured patients in the Netherlands. Eur J Trauma Emerg Surg. doi:10.1007/s00068-012-0223-9.
4. Walcher F, Weinlich M, Conrad G, Schweigkofler U, Breitkreutz R, Kirschning T, Marzi I. Prehospital ultrasound imaging improves management of abdominal trauma. Br J Surg. 2006;93:238-42.

5. Tajoddini S, Vahdati SS. Ultrasonographic diagnosis of abdominal free fluid: accuracy comparison of emergency physicians and radiologists. Eur J Trauma Emerg Surg. doi:10.1007/s00068-0120219-5.

6. Huber-Wagner S, Lefering R, Qvick L-M, Körner M, Kay MV, Pfeifer K-J, Reiser M, Mutschler W, Kanz K-G. Working Group on Polytrauma of the German Trauma Society: effect of wholebody CT during trauma resuscitation on survival: a retrospective, multicentre study. Lancet. 2009;373:1455-61.

7. Kepros JP, Opreanu RC, Samaraweera R, Briningstool A, Morrison CA, Mosher BD, Schneider P, Stevens P. Whole body imaging in the diagnosis of blunt trauma, ionizing radiation hazards and residual risk. Eur J Trauma Emerg Surg. doi:10.1007/ s00068-012-0201-2.

8. Dübendorfer C, Billeter AT, Seifert B, Keel M, Turina M. Serial lactate and admission SOFA scores in trauma: an analysis of predictive value in 724 patients with and without traumatic brain injury. Eur J Trauma Emerg Surg. doi:10.1007/s00068-012-0212-z. 